TRANSACTIONS OF THE

AMERICAN MATHEMATICAL SOCIETY

Volume 360, Number 12, December 2008, Pages 6475-6496

S 0002-9947(08)04463-2

Article electronically published on June 3, 2008

\title{
LAPLACE TRANSFORMS WHICH ARE NEGATIVE POWERS OF QUADRATIC POLYNOMIALS
}

\author{
G. LETAC AND J. WESOŁOWSKI
}

\begin{abstract}
We find the distributions in $\mathbb{R}^{n}$ for the independent random variables $X$ and $Y$ such that $\mathbb{E}(X \mid X+Y)=a(X+Y)$ and $\mathbb{E}(q(X) \mid X+Y)=$ $b q(X+Y)$ where $q$ runs through the set of all quadratic forms on $\mathbb{R}^{n}$ orthogonal to a given quadratic form $v$. The essential part of this class is provided by distributions with Laplace transforms $(1-2\langle c, s\rangle+v(s))^{-p}$ that we describe completely, obtaining a generalization of a Gindikin theorem. This leads to the classification of natural exponential families with the variance function of type $\frac{1}{p} m \otimes m-\varphi(m) M_{v}$, where $M_{v}$ is the symmetric matrix associated to the quadratic form $v$ and $m \mapsto \varphi(m)$ is a real function. These natural exponential families extend the classical Wishart distributions on Lorentz cones already considered by Jensen, and later on by Faraut and Korányi.
\end{abstract}

\section{INTRODUCTION}

As an attempt to extend the celebrated Lukacs' theorem on characterization of the gamma distribution (Lukacs, 1955), Wang (1981) observes the following interesting fact. Its statement uses the entire function

$$
f_{p}(z)=\sum_{m=0}^{\infty} \frac{z^{m}}{m ! \Gamma(m+p)},
$$

where $p>0$.

Theorem 1.1. Let $X=\left(X_{1}, X_{2}\right)$ and $Y=\left(Y_{1}, Y_{2}\right)$ be non-Dirac independent positive random variables valued in $\mathbb{R}^{2}$ such that there exist real numbers $a$ and $b$ with the following property:

$$
\mathbb{E}(X \mid X+Y)=a(X+Y), \mathbb{E}\left(X_{i}^{2} \mid X+Y\right)=b\left(X_{i}+Y_{i}\right)^{2}, \quad i=1,2 .
$$

Then $0<a^{2}<b<a<1$ and for $p=\frac{a^{2}-a b}{b-a^{2}}$ there exist non-negative numbers $a_{1}, a_{2}, a_{12}$ such that $a_{1} a_{2}-a_{12} \geq 0$, and for small enough $\left(s_{1}, s_{2}\right)$ one has

$$
\begin{aligned}
\mathbb{E}\left(e^{s_{1} X_{1}+s_{2} X_{2}}\right) & =\left(1-a_{1} s_{1}-a_{2} s_{2}+\left(a_{1} a_{2}-a_{12}\right) s_{1} s_{2}\right)^{-p} \\
\mathbb{E}\left(e^{s_{1} Y_{1}+s_{2} Y_{2}}\right) & =\left(1-a_{1} s_{1}-a_{2} s_{2}+\left(a_{1} a_{2}-a_{12}\right) s_{1} s_{2}\right)^{-\frac{1-a}{a} p} .
\end{aligned}
$$

Received by the editors May 8, 2006 and, in revised form, December 1, 2006.

2000 Mathematics Subject Classification. Primary 60E05, 44A10, 62E10.

Key words and phrases. Characterizations of probabilities, Gindikin Theorem, Lorentz cone, Wishart distributions, natural exponential families, variance functions. 
Furthermore if $a_{1} a_{2}-a_{12}>0$, the distribution of $X$ is

$$
\left(\theta_{1} \theta_{2}-1\right)^{p} e^{\theta_{1} x_{1}+\theta_{2} x_{2}} \frac{1}{\Gamma(p)}\left(x_{1} x_{2}\right)^{p-1} f_{p}\left(x_{1} x_{2}\right) \mathbf{1}_{(0, \infty)^{2}}\left(x_{1}, x_{2}\right) d x_{1} d x_{2},
$$

where $\theta_{1}=\frac{-a_{2}}{a_{1} a_{2}-a_{12}}$ and $\theta_{2}=\frac{-a_{1}}{a_{1} a_{2}-a_{12}}$.

The result is not difficult: just multiply (1.2) by $e^{s_{1}\left(X_{1}+Y_{1}\right)+s_{2}\left(X_{2}+Y_{2}\right)}$ and take expectations to obtain differential equations for the Laplace transforms of $X$ and $Y$.

A quasi-automatic way to extend Wang's result is to consider $X$ and $Y$ independent in $\mathbb{R}^{n}$ such that there exist $a$ and $b$ with

$$
\mathbb{E}(X \mid X+Y)=a(X+Y), \mathbb{E}\left(X_{i}^{2} \mid X+Y\right)=b\left(X_{i}+Y_{i}\right)^{2}, \quad i=1,2, \ldots, n .
$$

The same method of proof gives that there exists $p>0$ and coefficients $c_{T}$ with $T \subset\{1, \ldots, n\}$ such that the Laplace transform of $X$ has the form

$$
\left(\sum_{T} c_{T} s^{T}\right)^{-p}
$$

with the convention $s^{T}=\prod_{i \in T} s_{i}$. Special cases of these distributions occur in literature (see Bernardoff (2006), Bar-Lev et al. (1994), Griffiths (1984), and comment 4 in Section 6 below). Actually the set of acceptable parameters $\left(\left(c_{T}\right)_{T} ; p\right)$ is not known in general, and in most of the known cases, the density is unreachable.

A better way to extend Wang's bivariate characterization to $n$ dimensions is based on the following reformulation: let us observe that the second part of (1.2) can be rewritten by saying that

$$
\mathbb{E}(q(X) \mid X+Y)=b q(X+Y)
$$

for all quadratic forms on $\mathbb{R}^{2}$ of type $q(x)=\lambda_{1} x_{1}^{2}+\lambda_{2} x_{2}^{2}$ that are having a diagonal representative matrix $M_{q}=\left(\begin{array}{cc}\lambda_{1} & 0 \\ 0 & \lambda_{2}\end{array}\right)$. This two dimensional linear space of quadratic forms can be seen as the space of the quadratic forms on $\mathbb{R}^{2}$ which are orthogonal to the quadratic form $v(x)=2 x_{1} x_{2}$ whose representative matrix is $M_{v}=\left(\begin{array}{ll}0 & 1 \\ 1 & 0\end{array}\right)$. Here the space of quadratic forms has the scalar product $\left\langle q_{1}, q_{2}\right\rangle=\operatorname{tr} M_{q_{1}} M_{q_{2}}$ and orthogonality means $\operatorname{tr} M_{q_{1}} M_{q_{2}}=0$. Similarly, Bobecka and Wesolowski (2004) in their Theorem 3, instead of the second part of (1.2), consider the conditions $\mathbb{E}\left(X_{1}^{2} \mid X+Y\right)=b\left(X_{1}+Y_{1}\right)^{2}$ and $\mathbb{E}\left(X_{1} X_{2} \mid X+Y\right)=$ $b\left(X_{1}+Y_{1}\right)\left(X_{2}+Y_{2}\right)$. These conditions can be expressed by taking $q$ 's in (1.5) with the representative matrices of the form $M_{q}=\left(\begin{array}{cc}\lambda_{1} & \lambda_{2} \\ \lambda_{2} & 0\end{array}\right)$. These quadratic forms are orthogonal to $v$ where $M_{v}=\left(\begin{array}{ll}0 & 0 \\ 0 & 1\end{array}\right)$, namely $v(x)=x_{2}^{2}$. Our extension will be based on the consideration of (1.5) for $n \geq 2$ dimensions by taking all quadratic forms $q$ which are orthogonal to a fixed $v$ as in the previous two cases. The Laplace transforms of the distributions that we get are essentially of the form $(1-2\langle c, s\rangle+v(s))^{-p}$. The discussion is unfortunately somewhat obscured by consideration of several cases. But the heart of our method can be seen in formulas (2.13) to (2.15). This is covered in Section 2. 
In Section 3 we identify the acceptable triples $(c, v, p)$ for the Laplace transform of the form $(1-2\langle c, s\rangle+v(s))^{-p}$. We compute the corresponding probability distributions. This includes a generalization of the Gindikin Theorem (see Gindikin (1975)) about Wishart distributions on symmetric cones for the Lorentz cone (look at the comments after Theorem 3.1). Section 4 is devoted to a complete description by convolution semigroups of the exceptional case isolated by Bobecka and Wesołowski (2004).

After the point of view of regression illustrated by (1.5) (recall that statisticians use the term regression curve for the graph of the function $h(u)=\mathbb{E}(V \mid U=u)$ when $(U, V)$ is a vector random variable), after the second point of view of Laplace transforms which are negative powers of quadratic polynomials, a third one is given by natural exponential families $(\mathrm{NEF})$ on $\mathbb{R}^{n}$. We consider the NEF such that their variance function $V_{F}$ has the following property: for some $p>0$ the matrix

$$
V_{F}(m)-\frac{1}{p} m \otimes m
$$

is proportional to a constant symmetric matrix $M_{v}$ for all $m$. Surprisingly this condition is more or less equivalent to (1.5). The source of this point of view is the fact that when $M_{v}=\operatorname{diag}(1,-1, \ldots,-1)$, then

$$
V_{F}(m)=\frac{1}{p} m \otimes m+\frac{1}{4 p}\left(m_{1}^{2}-m_{2}^{2}-\ldots-m_{n}^{2}\right) M_{v}
$$

is the variance function of the NEF of the Wishart distributions on the Lorentz cone with shape parameter $p \geq(n-2) / 2$. This is the subject of Section 5 . Section 6 links the present paper with literature, specially on Euclidean Jordan algebras.

\section{Characterization By Quadratic Regression}

Here is our extension. As usual, $L_{X}(s)=\mathbb{E}\left(e^{\langle s, X\rangle}\right)$ denotes the Laplace transform of the random variable $X$.

Theorem 2.1. Let $n \geq 2$. Let $v$ be a quadratic form on $\mathbb{R}^{n}$ of rank $r$ and let $Q_{v}$ be the space of quadratic forms $q$ on $\mathbb{R}^{n}$ such that $\operatorname{tr}\left(M_{q} M_{v}\right)=0$. Let $X$ and $Y$ be non-Dirac independent random variables on $\mathbb{R}^{n}$ having exponential moments. Assume that there exists $a \in \mathbb{R}$ such that $\mathbb{E}(X \mid X+Y)=a(X+Y)$. Then $0<a<1$ and $L_{Y}=\left(L_{X}\right)^{(1-a) / a}$. Furthermore there exists a real number $b$ such that for all $q \in Q_{v}$

$$
\mathbb{E}(q(X) \mid X+Y)=b q(X+Y)
$$

if and only if $0<a^{2} \leq b \leq a<1$ and

1. Either $b=a$. In this case $r=1$. We write $v=\lambda e_{1} \otimes e_{1}$ for some vector $e_{1}$ of norm 1 and some real $\lambda \neq 0$. Then $X=e_{1} X^{\prime}$, where $X^{\prime}$ has an arbitrary distribution on the real line.

2. Or $b=a^{2}$. In this case $X$ has normal distribution in $\mathbb{R}^{n}$ with arbitrary mean, and covariance proportional to $v$, where $v$ or $-v$ is semi-positive definite.

3. Or $a^{2}<b<a$.

- Either $r \geq 2$, and there exist a real number $\lambda$ and $c \in \mathbb{R}^{n}$ such that for $p=\frac{a^{2}-a b}{b-a^{2}}$ one has

$$
\mathbb{E}\left(e^{\langle s, X\rangle}\right)=(1-2\langle c, s\rangle+\lambda v(s))^{-p} .
$$


- Or $r=1$. We write $v=\lambda e_{1} \otimes e_{1}$ for some vector $e_{1}$ of norm 1 and some real $\lambda \neq 0$. Then there exists $c$ in $\mathbb{R}^{n}$ orthogonal to $e_{1}$ and a real function $f_{1}$ defined on some open interval in $\mathbb{R}$ such that for $X=\left\langle X, e_{1}\right\rangle e_{1}+X^{\prime}$ one has

$$
\begin{aligned}
& \mathbb{E}\left(e^{\langle s, X\rangle}\right)=\mathbb{E}\left(e^{s_{1}\left\langle X, e_{1}\right\rangle+\left\langle s^{\prime}, X^{\prime}\right\rangle}\right)=\left(f_{1}\left(s_{1}\right)-\left\langle c, s^{\prime}\right\rangle\right)^{-p}, \\
& \text { where } s=s_{1} e_{1}+s^{\prime} \text { with }\left\langle s^{\prime}, e_{1}\right\rangle=0 .
\end{aligned}
$$

Proof. Since $X$ and $Y$ have exponential moments there exist two open maximal convex sets $\Theta(X)$ and $\Theta(Y)$ containing 0 on which their Laplace transforms $L_{X}(s)$ and $L_{Y}$ are finite. Denote their intersection by $\Theta$. We also write $\kappa_{X}=\log L_{X}$ and $\kappa_{Y}=\log L_{Y}$. Since $\mathbb{E}(X \mid X+Y)=a(X+Y)$ holds, let us multiply both sides by $e^{\langle s, X+Y\rangle}$ and take expectations. We get for $s$ in $\Theta$ :

$$
(1-a) L_{X}^{\prime}(s) L_{Y}(s)=a L_{X}(s) L_{Y}^{\prime}(s)
$$

that we rewrite as $(1-a) \kappa_{X}^{\prime}=a \kappa_{Y}^{\prime}$. Since $X$ and $Y$ are non-Dirac, $a=0$ and $a=1$ are impossible. Since $\kappa_{X}$ and $\kappa_{Y}$ are convex, $a$ and $(1-a)$ have the same sign and $a \in(0,1)$. Since $\kappa_{X}(0)=\kappa_{Y}(0)=0$ we get $L_{Y}(s)=\left(L_{X}(s)\right)^{(1-a) / a}$ on $\Theta$ and by the principle of maximal analyticity for Laplace transforms we get $\Theta(X)=\Theta(Y)=\Theta$. For simplicity we now denote $\kappa=\kappa_{X}$.

We use the following notation: if $P(s)=\sum_{\alpha \in \mathbb{N}^{n}} p_{\alpha} s_{1}^{\alpha_{1}} \ldots s_{n}^{\alpha_{n}}$ is any real polynomial with respect to $n$ variables, we denote by $P\left(\frac{\partial}{\partial s}\right)$ the differential operator

$$
P\left(\frac{\partial}{\partial s}\right)=\sum_{\alpha \in \mathbb{N}^{n}} p_{\alpha}\left(\frac{\partial}{\partial s_{1}}\right)^{\alpha_{1}} \ldots\left(\frac{\partial}{\partial s_{n}}\right)^{\alpha_{n}} .
$$

In particular if $f$ is any $C^{2}$ real function of $n$ variables with gradient $f^{\prime}$ and Hessian $f^{\prime \prime}$ and if $q$ is a quadratic form, note that we can write $q\left(\frac{\partial}{\partial s}\right)(f)=\operatorname{tr}\left(M_{q} f^{\prime \prime}\right)$ and $q\left(f^{\prime}\right)=\operatorname{tr}\left(M_{q}\left(f^{\prime} \otimes f^{\prime}\right)\right)$.

The next equality is again rather standard. For $s \in \Theta$ we multiply both sides of (2.6) by $e^{\langle s, X+Y\rangle}$ and take expectations. We get for all $q \in Q_{v}$

$$
\left(1-\frac{b}{a}\right) q\left(\frac{\partial}{\partial s}\right) \kappa=\left(\frac{b}{a^{2}}-1\right) q\left(\kappa^{\prime}\right),
$$

since in general we have for any quadratic form $q$ on $\mathbb{R}^{n}$

$$
q\left(\frac{\partial}{\partial s}\right) e^{\kappa(s)}=e^{\kappa(s)}\left(q\left(\frac{\partial}{\partial s}\right) \kappa(s)+q\left(\kappa^{\prime}(s)\right)\right)
$$

and

$$
q\left(\frac{\partial}{\partial s}\right)(f g)(s)=g(s) q\left(\frac{\partial}{\partial s}\right) f(s)+2 q\left(f^{\prime}(s), g^{\prime}(s)\right)+f(s) q\left(\frac{\partial}{\partial s}\right) g(s)
$$

with the notation $q(x, y)=\frac{1}{2}(q(x+y)-q(x)-q(y))$ for the polarized form of $q$. We now discuss the solutions of (2.9) in the various cases $b=a, b=a^{2}, a^{2}<b<a$, and we will show after that $b \notin\left[a^{2}, a\right]$ is impossible.

THE CASE $b=a$.

From (2.9) we can claim that $q\left(\kappa^{\prime}(s)\right)=0$ for all $q \in Q_{v}$ and for all $s \in \Theta$. Suppose first that $v=0$. Then $Q_{v}$ is the set of all quadratic forms: taking $q$ positive definite implies $\kappa^{\prime}(s)=0$ and $X$ Dirac, a case that we have excluded. Suppose that $v \neq 0$. Then $q\left(\kappa^{\prime}(s)\right)=0$ can be reformulated as $\operatorname{tr}\left[M_{q}\left(\kappa^{\prime}(s) \otimes \kappa^{\prime}(s)\right)\right]=0$. Since this is true for any $q \in Q_{v}$, this implies the existence of a scalar function $f(s)$ such 
that $\kappa^{\prime}(s) \otimes \kappa^{\prime}(s)=f(s) v$. Since $X$ is non-Dirac, there exists $s_{0}$ such that $\kappa^{\prime}\left(s_{0}\right) \neq 0$ and thus $v$ has rank 1 . Without loss of generality, by changing the orthonormal basis into a new basis $\left(e_{1}, \ldots, e_{n}\right)$ if necessary, we assume that $M_{v}$ is written by blocks as $M_{v}=\left[\begin{array}{ll}1 & 0 \\ 0 & 0\end{array}\right]$, where the square diagonal blocks are of order 1 and $n-1$, respectively. Thus

$$
\left(\frac{\partial \kappa}{\partial s_{1}}\right)^{2}=f(s), \frac{\partial \kappa}{\partial s_{j}}=0, j=2, \ldots, n .
$$

Thus $X$ is concentrated on the line $\mathbb{R} e_{1}$. This leads to the result.

THE CASE $b=a^{2}$.

By (2.9) we get that $q\left(\frac{\partial}{\partial s}\right) \kappa(s)=0$ for all $s$. Furthermore this is true for all $q \in Q_{v}$. Thus there exists a scalar function $s \mapsto g(s)$ on $\Theta$ such that $\kappa^{\prime \prime}(s)=g(s) v$. We show that $g$ is a constant function. To see this, without loss of generality by changing the orthonormal basis if necessary, we assume that $M_{v}=\left(v_{i j}\right)$ is diagonal. Thus we can write for any $i, j, l$ in $\{1, \ldots, n\}$

$$
\frac{\partial^{3} \kappa}{\partial s_{i} \partial s_{j} \partial s_{l}}=\frac{\partial g}{\partial s_{l}} v_{i j}=\frac{\partial g}{\partial s_{i}} v_{l j}=\frac{\partial g}{\partial s_{j}} v_{i l} \text {. }
$$

Since $n \geq 2$, by choosing $i=j \neq l$ we easily deduce from this that $\frac{\partial g}{\partial s_{i}}=0$ for all $i$. Thus $X$ is Gaussian as indicated, and $v$ or $-v$ has to be semipositive definite.

THe CASE $a^{2}<b<a$.

We introduce the positive number $p=\frac{a^{2}-a b}{b-a^{2}}$ such that (2.9) becomes $p q\left(\frac{\partial}{\partial s}\right) \kappa=$ $q\left(\kappa^{\prime}\right)$ which can be rewritten

$$
\operatorname{tr}\left(M_{q}\left(p \kappa^{\prime \prime}-\kappa^{\prime} \otimes \kappa^{\prime}\right)\right)=0
$$

Since this holds true for all $q \in Q_{v}$, this is equivalent to the existence of a scalar function $g$ on $\Theta$ such that

$$
\kappa^{\prime \prime}(s)-\frac{1}{p} \kappa^{\prime}(s) \otimes \kappa^{\prime}(s)=g(s) v .
$$

THE SUBCASE $a^{2}<b<a$ AND $2 \leq r$.

We assume that $r \geq 2$. We prove first that either $g$ has no zeros, or $g$ is identically 0 . If $g\left(s_{0}\right)=0$ for some $s_{0} \in \Theta$, by changing the orthonormal basis if necessary we assume that $\kappa^{\prime}\left(s_{0}\right)$ is a multiple of $e_{1}$, where $e=\left(e_{1}, \ldots, e_{n}\right)$ is the canonical basis of $\mathbb{R}^{n}$. Thus $\kappa^{\prime \prime}\left(s_{0}\right)$ is proportional to the block matrix $\left[\begin{array}{ll}1 & 0 \\ 0 & 0\end{array}\right]$, where the diagonal blocks are square matrices of order 1 and $n-1$, respectively. Since $\kappa^{\prime \prime}\left(s_{0}\right)$ is the covariance of the probability $e^{\left\langle s_{0}, x\right\rangle-\kappa\left(s_{0}\right)} \mu(d x)$ where $X \sim \mu$, this implies that $\mu$ is concentrated on $\mathbb{R} e_{1}$, thus $\kappa^{\prime \prime}(s)$ is proportional to $\left[\begin{array}{ll}1 & 0 \\ 0 & 0\end{array}\right]$ for all $s$, which implies that $g(s)=0$ for all $s \in \Theta$. Thus $\kappa$ is a function of $s_{1}$ alone and we get

$$
\frac{\partial^{2} \kappa}{\partial s_{1}^{2}}=\frac{1}{p}\left(\frac{\partial \kappa}{\partial s_{1}}\right)^{2}
$$


This easily shows that $X_{1}$ is gamma distributed with the shape parameter $p$. This provides formula (2.7) for the case $\lambda=0$.

We now assume that $g$ has no zeros on $\Theta$. Thus we denote $f=-1 / g$. We now rewrite

$$
\kappa^{\prime \prime}(s)=\frac{1}{p} \kappa^{\prime}(s) \otimes \kappa^{\prime}(s)-\frac{1}{f(s)} v
$$

as an equality between two symmetric bilinear forms on $\mathbb{R}^{n}$ with respect to the variables $h_{1}$ and $h_{2}$ of $\mathbb{R}^{n}$. We obtain

$$
\kappa^{\prime \prime}(s)\left(h_{1}, h_{2}\right)=\frac{1}{p} \kappa^{\prime}(s)\left(h_{1}\right) \kappa^{\prime}(s)\left(h_{2}\right)-\frac{1}{f(s)} v\left(h_{1}, h_{2}\right) .
$$

We now take the differential of both sides of (2.12) and estimate it in the direction $h_{3}$ of $\mathbb{R}^{n}$. For simplification, write $a_{i}=\kappa^{\prime}(s)\left(h_{i}\right)=\left\langle\kappa^{\prime}(s), h_{i}\right\rangle$. We get the equality

$$
\begin{aligned}
\kappa^{\prime \prime \prime}(s)\left(h_{1}, h_{2}, h_{3}\right)= & \frac{1}{p} a_{1} \kappa^{\prime \prime}(s)\left(h_{2}, h_{3}\right)+\frac{1}{p} a_{2} \kappa^{\prime \prime}(s)\left(h_{1}, h_{3}\right)+\frac{\left\langle f^{\prime}(s), h_{3}\right\rangle}{f(s)^{2}} v\left(h_{1}, h_{2}\right) \\
= & \frac{2}{p^{2}} a_{1} a_{2} a_{3}-\frac{1}{p f(s)}\left(a_{1} v\left(h_{2}, h_{3}\right)+a_{2} v\left(h_{1}, h_{3}\right)+a_{3} v\left(h_{1}, h_{2}\right)\right) \\
& +\left(\frac{a_{3}}{p f(s)}+\frac{\left\langle f^{\prime}(s), h_{3}\right\rangle}{f(s)^{2}}\right) v\left(h_{1}, h_{2}\right)
\end{aligned}
$$

(we have transformed (2.13) with the help of (2.12)). Now since (2.13) and (2.14) are trilinear symmetric forms in $\left(h_{1}, h_{2}, h_{3}\right)$, this implies that (2.15) is also a trilinear symmetric form.

Since $\left(h_{1}, h_{2}\right) \mapsto v\left(h_{1}, h_{2}\right)$ is not of rank 0 or 1 , the fact that (2.15) is a trilinear symmetric form implies that the linear form

$$
h_{3} \mapsto \frac{a_{3}}{p f(s)}+\frac{\left\langle f^{\prime}(s), h_{3}\right\rangle}{f(s)^{2}}
$$

is zero, that is, $f(s) \kappa^{\prime}(s)+p f^{\prime}(s)=0$. Taking the differential of this equality and using (2.12) gives $f^{\prime \prime}(s)=\frac{1}{p} v$. This implies the existence of constants $\alpha \in \mathbb{R}$ and $\beta \in \mathbb{R}^{n}$ such that $f(s)=\alpha+\langle\beta, s\rangle+\frac{1}{2 p} v(s)$. Now the equality $f(s) \kappa^{\prime}(s)+p f^{\prime}(s)=0$ gives that $\kappa(s)=-p \log (f(s) / \alpha$ ) since $\kappa(0)=0$ (note that $f$ is never zero on $\Theta$ and thus $\alpha=f(0) \neq 0)$. Thus there exists $\lambda=\frac{1}{2 p \alpha} \neq 0$ and $c=-\frac{\beta}{2 \alpha} \in \mathbb{R}^{n}$ such that $\mathbb{E}\left(e^{\langle s, X\rangle}\right)=(1-2\langle c, s\rangle+\lambda v(s))^{-p}$. For further use in Section 5 , note that

$$
f(s)=\frac{\lambda}{2 p}(1-2\langle c, s\rangle+\lambda v(s)) .
$$

THE SUBCASE $a^{2}<b<a$ AND $r=1$.

If $g$ is identically zero on $\Theta$ we are sent back to (2.10) as in the case $r \geq 2$. In this case, (2.8) holds with $c=0$ and an affine $f_{1}$ which is the same as (2.7) with $\lambda=0$.

If $g$ is not identically zero, denote

$$
\Theta_{0}=\{s \in \Theta ; g(s) \neq 0\} .
$$

Since $g$ is analytic, $\Theta_{0}$ is an open set and we define $f=-1 / g$ on $\Theta_{0}$.

We write $v(s)= \pm\langle w, s\rangle^{2}$ for some non-zero vector $w \in \mathbb{R}^{n}$. We take without loss of generality $w$ as the unit vector $e_{1}$ (by doing a suitable linear transformation), 
and we write $s=s_{1} e_{1}+\ldots+s_{n} e_{n}$ where $\left(e_{1}, \ldots, e_{n}\right)$ is the standard basis of $\mathbb{R}^{n}$. We rewrite (2.11) suitably expressed in coordinates as

$$
\begin{aligned}
\frac{\partial^{2} \kappa(s)}{\partial s_{1}^{2}} & =\frac{1}{p}\left(\frac{\partial \kappa(s)}{\partial s_{1}}\right)^{2}-\frac{1}{f(s)} \\
\frac{\partial^{2} \kappa(s)}{\partial s_{i} \partial s_{j}} & =\frac{1}{p} \frac{\partial \kappa(s)}{\partial s_{i}} \frac{\partial \kappa(s)}{\partial s_{j}} \text { if }(i, j) \neq(1,1) .
\end{aligned}
$$

First differentiate (2.17) with respect to $s_{j}$ with $j \neq 1$. In (2.18) take $i=1$ and differentiate with respect to $s_{1}$. Taking the difference between these two equalities makes that the third derivatives disappear. Plugging in the resulting equation formulas (2.17) and (2.18) we get for $j \neq 1$ :

$$
\frac{1}{p} \frac{\partial \kappa(s)}{\partial s_{j}}=-\frac{1}{f(s)} \frac{\partial f(s)}{\partial s_{j}} .
$$

Now differentiate (2.19) with respect to $s_{i}, i=1, \ldots, n$, and using again (2.18) and (2.19) we get for $(i, j) \neq(1,1)$ :

$$
\frac{\partial^{2} f(s)}{\partial s_{i} \partial s_{j}}=0 .
$$

Since $\Theta_{0}$ is not empty, it contains an open cube $\Theta_{1}=\left(u_{1}, v_{1}\right) \times \ldots \times\left(u_{n}, v_{n}\right)$ and there exists a real function $s_{1} \mapsto C_{1}\left(s_{1}\right)$ on $\left(u_{1}, v_{1}\right)$ and constants $C_{2}, \ldots, C_{n}$ such that for all $s$ in $\Theta_{1}$ we have

$$
f(s)=C_{1}\left(s_{1}\right)+C_{2} s_{2}+\ldots+C_{n} s_{n} .
$$

Furthermore, by definition, $f$ is never zero on $\Theta_{0}$, and without loss of generality we may assume that $f>0$ on $\Theta_{1}$ (if not, we change $v$ into $-v$ ). Here we have used the fact that $\Theta_{1}$ is connected.

On the other hand integrating (2.19) leads to the existence of functions $D_{j}(s)$ on $\Theta_{1}$ such that $D_{j}$ does not depend on $s_{j}$ and such that for any $j=2, \ldots, n$, we have $\kappa(s)=-p \log f(s)+D_{j}(s)$. All the $D_{j}$ 's are therefore equal to a common function $s_{1} \mapsto D\left(s_{1}\right)$ defined on $\left(u_{1}, v_{1}\right)$, and thus

$$
\kappa(s)=-p \log f(s)+D\left(s_{1}\right) .
$$

We carry (2.22) into (2.18) taken for $(i, j)=(1,2)$. After cancellation with the help of (2.20) we find that $D$ is a constant. Finally writing $c_{j}=-C_{j} e^{-D / p}$ for $j=2, \ldots, n$ and $f_{1}\left(s_{1}\right)=C_{1}\left(s_{1}\right) e^{-D / p}$, we get (2.8) on $\Theta_{1}$. By the principle of maximal analyticity for Laplace transforms, we conclude that (2.8) holds on $\Theta$ itself.

The CASE $b \notin\left[a^{2}, a\right]$.

The last thing to prove is to check that $b \notin\left[a^{2}, a\right]$ is impossible. The algebraic calculations of the case $a^{2}<b<a$ are still valid, but with $p<0$. If $r \geq 2$ we end up with the Laplace transform $(1-2\langle c, s\rangle+\lambda v(s))^{-p}$. We discuss the case $\lambda \neq 0$. Since $Q_{v}=Q_{\lambda v}$, we replace $\lambda v$ by $v$. Without loss of generality we can also choose the coordinates such that $v(s)=s_{1}^{2}+s_{2}^{2}+\ldots+s_{k}^{2}-s_{k+1}^{2}-\ldots-s_{r}^{2}$, where $0 \leq k \leq r \leq n$. Then

$1-2\langle c, s\rangle+v(s)=1-2 c_{1} s_{1}-\ldots-2 c_{r} s_{r}-\ldots-2 c_{n} s_{n}+s_{1}^{2}+s_{2}^{2}+\ldots+s_{k}^{2}-s_{k+1}^{2}-\ldots-s_{r}^{2}$. 
This shows that for $j>r$ the second derivative of the convex function $s_{j} \mapsto$ $-p \log (1-2\langle c, s\rangle+\lambda v(s))$ is $4 p c_{j}^{2}(1-2\langle c, s\rangle+\lambda v(s))^{2}$. Since $p<0$ this implies $c_{j}=0$.

Thus without loss of generality we assume $r=n$. Equality (2.11) applied to the case $p<0$ shows that either $k=0$ or $k=n$ : this comes from the fact that

$$
\kappa^{\prime \prime}(s)+\frac{1}{|p|} \kappa^{\prime}(s) \otimes \kappa^{\prime}(s)=\frac{1}{f(s)} v
$$

is necessarily positive definite as the sum of a positive definite operator $\kappa^{\prime \prime}(s)$ and of a semipositive definite one. Write $\epsilon=-1$ for $k=0$ and $\epsilon=1$ for $k=n$. Furthermore we see that $1-2\langle c, s\rangle+v(s)=a+v(s-\epsilon c)$ where $a=1-v(c)$. If $\mu(d x)$ is the probability with Laplace transform $(1-2\langle c, s\rangle+v(s))^{-p}$ defined on an open convex set $\Theta$ containing 0 , consider the possibly unbounded measure $e^{\langle\epsilon c, x\rangle} \mu(d x)$ with Laplace transform $(a+v(s))^{-p}$ defined on $\Theta+\epsilon c$. Renormalization according to $a$ and discussion according to the facts that either $v$ or $-v$ is positive definite and that $a<0, a=0, a>0$ leads us to consider the four cases

$$
\begin{aligned}
& \left(1-s_{1}^{2}-\ldots-s_{n}^{2}\right)^{-p}, \\
& \left(1+s_{1}^{2}+\ldots+s_{n}^{2}\right)^{-p}, \\
& \left(s_{1}^{2}+\ldots+s_{n}^{2}\right)^{-p} \\
& \left(-1+s_{1}^{2}+\ldots+s_{n}^{2}\right)^{-p} .
\end{aligned}
$$

None of these 4 functions $\psi(s)$ can be the Laplace transform of some positive measure on $\mathbb{R}^{n}$. Because if it is the case, then for a fixed $s_{0} \in \mathbb{R}^{n} \backslash\{0\}$ the function $t \mapsto \psi\left(s_{0} t\right)$ defined on some interval of the real line would be the Laplace transform of a positive measure on $\mathbb{R}$. But $t \mapsto \log \psi\left(t s_{0}\right)$ is never convex. We finally treat the case $\lambda=0$ in a similar way. Thus the case $r \geq 2$ is settled.

If $r=1$ we end up with a Laplace transform $L\left(s_{1}\right)\left(1-C\left(s_{1}\right) s_{2}\right)^{-p}$ (after some changing of coordinates). Since the logarithm of $s_{2} \mapsto L\left(s_{1}\right)\left(1-C\left(s_{1}\right) s_{2}\right)^{-p}$ is not convex for $p<0$ the proof of the direct part of Theorem 2.1 is complete. The converse statements are easily obtained by reversing the above process.

$$
\text { 3. LAPLACE TRANSFORMS }(1-2\langle c, s\rangle+v(s))^{-p}
$$

In this section we will describe distributions $\mu=\mu_{p, c, v}$ on $\mathbb{R}^{n}$ whose Laplace transforms on a certain open convex set $\Theta$ containing 0 are of the form $(1-2\langle c, s\rangle+v(s))^{-p}$, where $v$ is a quadratic form of rank $r \leq n, c \in \mathbb{R}^{n}$ and $p>0$. These distributions occur in Theorem 2.1 with $\lambda v$ replacing $v$. However since $Q_{v}=Q_{\lambda v}$ for $\lambda \neq 0$ we may assume $\lambda=1$.

Before this, we recall a famous result due to Gindikin (1975): there exists a positive measure $\nu_{p}$ on $\mathbb{R}^{n}$ such that for $-\theta$ in the open Lorentz cone

$$
\Omega=\left\{x \in \mathbb{R}^{n} ; x_{1}>\left(x_{2}^{2}+\ldots+x_{n}^{2}\right)^{1 / 2}\right\}
$$

one has

$$
\int e^{\langle\theta, x\rangle} \nu_{p}(d x)=\left(\theta_{1}^{2}-\left(\theta_{2}^{2}+\ldots+\theta_{n}^{2}\right)\right)^{-p}
$$

if and only if $p \geq(n-2) / 2$. Under these circumstances for $p>(n-2) / 2$ the measure $\nu_{p}$ is concentrated on $\Omega$ and is equal to

$$
\nu_{p}(d x)=\left(x_{1}^{2}-x_{2}^{2}-\ldots-x_{n}^{2}\right)^{p-\frac{n}{2}} \frac{d x}{2^{2 p-\frac{n}{2}} \Gamma_{\Omega}(p)}
$$


where $\Gamma_{\Omega}(p)=(2 \pi)^{\frac{n-2}{2}} \Gamma(p) \Gamma\left(p-\frac{n-2}{2}\right)$. For $p=(n-2) / 2$ the measure $\nu_{p}$ is the image of the Lebesgue measure on $\mathbb{R}^{n-1}$ by $\left(x_{2}, \ldots, x_{n}\right) \mapsto\left(\left(x_{2}^{2}+\ldots+x_{n}^{2}\right)^{1 / 2}, x_{2}, \ldots, x_{n}\right)$, up to a suitable multiplicative constant and is thus concentrated on the boundary of $\Omega$.

Next, if $\rho$ is a non-negative number and if $p$ is still $\geq(n-2) / 2$ we use $\nu_{p}(d x)$ to build a more general measure $\nu_{p, \rho}$ on the closure $\bar{\Omega}$ of Lorentz cone (3.23). It is defined by

$$
\nu_{p, \rho}=\sum_{j=0}^{\infty} p(p+1) \ldots(p+j-1) \frac{\rho^{j}}{j !} \nu_{p+j}
$$

where $\nu_{p}$ is defined in (3.24). By the Newton binomial theorem

$$
(1-z)^{-p}=\sum_{j=0}^{\infty} p(p+1) \ldots(p+j-1) \frac{z^{j}}{j !}
$$

correct for $|z|<1$ and applied to $z=\rho /\left(\theta_{1}^{2}-\left(\theta_{2}^{2}+\ldots+\theta_{n}^{2}\right)\right)$, the Laplace transform of $\nu_{p, \rho}$ is defined on $-\Omega_{\rho}$ where

$$
\Omega_{\rho}=\left\{\theta \in \mathbb{R}^{n} ; \theta_{1}>\left(\theta_{2}^{2}+\ldots+\theta_{n}^{2}+\rho\right)^{1 / 2}\right\}
$$

and is

$$
\left(\theta_{1}^{2}-\theta_{2}^{2}-\ldots-\theta_{n}^{2}-\rho\right)^{-p}
$$

Note that $\nu_{p, 0}=\nu_{p}$ and that $\Omega_{0}=\Omega$. If $p>(n-2) / 2$ the explicit form of $\nu_{p}$ given in (3.24) leads to the relatively explicit form

$$
\nu_{p, \rho}(d x)=\frac{[v(x)]^{p-\frac{n}{2}}}{2^{2 p-\frac{n}{2}}(2 \pi)^{\frac{n-2}{2}} \Gamma_{\Omega}(p)} f_{p-\frac{n-2}{2}}\left(\frac{\rho v(x)}{4}\right) \mathbf{1}_{\Omega}(x) d x
$$

where the function $f_{p}$ is defined by (1.1) and where $v(x)=x_{1}^{2}-x_{2}^{2}-\ldots-x_{n}^{2}$. If $p=(n-2) / 2$ the measure $\nu_{p, \rho}$ contains the singular part $\nu_{\frac{n-2}{2}}$ and an absolutely continuous part. It is

$$
\nu_{\frac{n-2}{2}, \rho}(d x)=\nu_{\frac{n-2}{2}}(d x)+\frac{\rho}{2^{\frac{n}{2}}(2 \pi)^{\frac{n-2}{2}} \Gamma\left(\frac{n-2}{2}\right)} f_{2}\left(\frac{\rho v(x)}{4}\right) \mathbf{1}_{\Omega}(x) d x .
$$

We skip the proofs of (3.26) and of (3.27), which are easily done by checking either the densities or the Laplace transforms of both sides.

In order to state the theorem, we introduce a notation. Given a quadratic form $v$ on a Euclidean space $E$ with rank $r \leq \operatorname{dim} E=n$ we associate to $v$ a symmetric endomorphism $s_{v}$ of $E$ defined by $\left\langle s_{v}(x), x\right\rangle=v(x)$. Its image $F$ has dimension $r$, and the restriction of $s_{v}$ is an automorphism of $F$ which has an inverse. Therefore we define a quadratic form $v^{-1}$ on $F$ by $\left\langle s_{v}^{-1}(x), x\right\rangle=v^{-1}(x)$. For instance if $E=\mathbb{R}^{n}$ has its natural Euclidean structure, a suitable change of orthonormal basis gives $v(x)=\lambda_{1} x_{1}^{2}+\ldots+\lambda_{k} x_{k}^{2}-\lambda_{k+1} x_{k+1}^{2}-\ldots-\lambda_{r} x_{r}^{2}$ with $\lambda_{j}>0$ and $r \leq n$. Then $F=\mathbb{R}^{r} \times\{0\}$ and

$$
v^{-1}(x)=\frac{x_{1}^{2}}{\lambda_{1}}+\ldots+\frac{x_{k}^{2}}{\lambda_{k}}-\frac{x_{k+1}^{2}}{\lambda_{k+1}}-\ldots-\frac{x_{r}^{2}}{\lambda_{r}}
$$

If $c=\left(c_{1}, \ldots, c_{n}\right)$ in this new system of coordinates, then the projection $c_{F}$ of $c$ on the space $F$ is $c_{F}=\left(c_{1}, \ldots, c_{r}, 0, \ldots, 0\right)$. 
Theorem 3.1. Let $p>0$, let $c \in \mathbb{R}^{n}$ and let $v$ be a quadratic form on $\mathbb{R}^{n}$ with signature $(k, r-k, n-r)$ (that is, having, respectively, $k$ plus signs, $r-k$ minus signs and $n-r$ zeros in the Sylvester decomposition). We write $c_{F}$ as the orthogonal projection of $c$ on the image $F$ of the symmetric endomorphism $s_{v}$, and we write $\rho=v^{-1}\left(c_{F}\right)-1$. Denote by $\mu_{p, c, v}$ the probability on $\mathbb{R}^{n}$ with Laplace transform $(1-2\langle c, s\rangle+v(s))^{-p}$. Then $\mu_{p, c, v}$ exists if and only if the following circumstances are met:

1. $k=0$. In this case, $-v$ is semipositive definite and $\mu_{p, c, v}$ is the distribution of the random vector

$$
X T^{1 / 2}+T c,
$$

where $X$ and $T$ are independent, $X$ is centered and normally distributed in $\mathbb{R}^{n}$ with covariance $-v$ and $T$ is a gamma variable $\gamma_{p, 2}$.

2. $k=1=n, p>0$ and $c_{1}^{2} \geq 1$. If $c_{1}=\epsilon \cosh \theta$ with $\epsilon= \pm 1$ let $T_{1} \sim \gamma_{p, e^{\theta}}$ and $T_{2} \sim \gamma_{p, e^{\theta}}$ be independent. With this notation $\mu_{p, c, v}$ is the distribution of $\epsilon\left(T_{1}+T_{2}\right)$.

3. $k=1<n,(n-2) / 2 \leq p$ and $\rho \geq 0$. Changing the coordinates we may assume that $v(x)=x_{1}^{2}-x_{2}^{2}-\ldots-x_{n}^{2}$ and $c_{1}>0$. With this notation

$$
\mu_{p, c, v}(d x)=e^{-c_{1} x_{1}+c_{2} x_{2}+\ldots+c_{n} x_{n}} \nu_{p, \rho}(d x) .
$$

Comments. Note that this statement offers a generalization of the Gyndikin Theorem, by showing that if $\rho \geq 0$ the function defined on $-\Omega_{\rho}$ (see (3.25) by $\left(\theta_{1}^{2}-\theta_{2}^{2}-\ldots-\theta_{n}^{2}-\rho\right)^{-p}$ is the Laplace transform of a positive measure if and only if $p \geq(n-2) / 2$.

To get an intuitive feeling of the probability (3.29) in case 3, observe that for $\rho=0$ it is the classical Wishart distribution on the closed Lorentz cone $\bar{\Omega}$. Now, for $\rho>0$, define $R=1-(1+\rho)^{1 / 3}, c_{0}=(1-R) c$ and $v_{0}=(1-R) v$. This choice of $R$ implies $\rho_{0}=v_{0}\left(c_{0}\right)-1=0$. Now consider the random variable $(X, J)$ valued in $\bar{\Omega} \times \mathbb{N}$ such that the distribution of the integer valued random variable $J$ is the negative binomial distribution

$$
\left(\delta_{0}+\sum_{j=1}^{\infty} \frac{p(p+1) \ldots(p+j-1)}{j !} R^{j} \delta_{j}\right)(1-R)^{p}
$$

and such that the distribution of $X$ conditioned by $J=j$ is the Wishart distribution on the closed Lorentz cone $\mu_{p+j, c_{0}, v_{0}}(d x)$. Then routine calculation of the Laplace transform of $X$ shows that $X \sim \mu_{p, c, v}$.

Proof. Given $v$ with the signature $(k, r-k, n-r)$ we first choose orthonormal coordinates such that $v(s)=\lambda_{1} s_{1}^{2}+\ldots+\lambda_{k} s_{k}^{2}-\lambda_{k+1} s_{k+1}^{2}-\ldots-\lambda_{r} s_{r}^{2}$, where $\lambda_{j}>0$ for $j=1, \ldots, r$. If $\left(X_{1}, \ldots, X_{n}\right)$ is a random variable having Laplace transform $(1-2\langle c, s\rangle+v(s))^{-p}$, then $\left(Y_{1}, \ldots, Y_{n}\right)=\left(\lambda_{1}^{-1 / 2} X_{1}, \ldots, \lambda_{r}^{-1 / 2} X_{r}, X_{r+1}, \ldots, X_{n}\right)$ has Laplace transform $\left(1-2\left\langle c^{\prime}, s\right\rangle+v^{\prime}(s)\right)^{-p}$, with $v^{\prime}(s)=s_{1}^{2}+\ldots+s_{k}^{2}-s_{k+1}^{2}-\ldots-s_{r}^{2}$ and with $c_{j}^{\prime}=\lambda_{1}^{-1 / 2} c_{j}$ for $j \leq r$ and $c_{j}^{\prime}=c_{j}$ for $j>r$. Note that $v^{\prime}\left(c^{\prime}\right)=v^{-1}\left(c_{F}\right)$. Thus from now on in the remainder of the proof of the present theorem we assume that

$$
v(s)=s_{1}^{2}+\ldots+s_{k}^{2}-s_{k+1}^{2}-\ldots-s_{r}^{2} .
$$


Note that the Hessian matrix $v^{\prime \prime}(s)$ is constant and equal to $M_{v}$. Since the convex function $\kappa(s)=-p \log (1-2\langle c, s\rangle+v(s))$ has second derivative

$\kappa^{\prime \prime}(s)=\frac{p}{(1-2\langle c, s\rangle+v(s))^{2}}\left[\left(2 c-v^{\prime}(s)\right) \otimes\left(2 c-v^{\prime}(s)\right)-(1-2\langle c, s\rangle+v(s)) v^{\prime \prime}(s)\right]$,

this implies that $k=0$ or 1 . To see this, observe that $k \geq 2$ would imply that the restriction of the above quadratic form to $s_{1}, \ldots, s_{k}$ is not positive definite: the sum of a quadratic form containing at least two negative signs in its signature and of a quadratic form of rank one cannot be positive definite.

THE CASE $k=0$. Writing

$$
\frac{1}{\left(1-2\langle c, s\rangle-s_{1}^{2}-\ldots-s_{r}^{2}\right)^{p}}=\int_{0}^{\infty} e^{-t\left(1-2\langle c, s\rangle-s_{1}^{2}-\ldots-s_{r}^{2}\right)} t^{p-1} \frac{d t}{\Gamma(p)},
$$

the existence and the probabilistic interpretation of the corresponding distribution $\mu$ is as follows: suppose that $X$ is standard normal in $\mathbb{R}^{r}$ and is considered as a variable in $\mathbb{R}^{n}$ concentrated on $\mathbb{R}^{r} \times\{0\}$. If $T$ is a gamma variable $\gamma_{p, 2}$ independent of $X$, then the distribution $\mu_{p, c, v}$ of

$$
X T^{1 / 2}+c T
$$

has the above Laplace transform.

THE CASE $k=n=1$. First we observe that $k=n=1$ implies that $c_{1}^{2} \geq 1$. Indeed $c_{1}^{2}<1$ implies that $1-2 c_{1} s_{1}+s_{1}^{2}$ has no real root. Thus the Laplace transform is defined on the whole line, but computation shows that its logarithm $\kappa$ satisfies $\kappa^{\prime \prime}(c)<0$. If $c_{1}^{2}=\cosh ^{2} \theta \geq 1$ with $\epsilon= \pm 1$ being the sign of $c_{1}$, then

$$
\frac{1}{\left(1-2 c_{1} s_{1}+s_{1}^{2}\right)^{p}}=\frac{1}{\left(1-s_{1} \epsilon e^{\theta}\right)^{p}} \frac{1}{\left(1-s_{1} \epsilon e^{-\theta}\right)^{p}},
$$

and $\mu_{p, c, v}$ is clearly the convolution of two gamma distributions $\gamma_{p, e^{\theta}}$ and $\gamma_{p, e^{-\theta}}$ when $\epsilon=1$ and symmetric of this one when $\epsilon=-1$.

The CASE $k=1$ AND $r=n \geq 2$. The case $k=n=1$ has shown $c_{1}^{2} \geq 1$. We denote

$$
\rho=c_{1}^{2}-c_{2}^{2}-\ldots-c_{n}^{2}-1 .
$$

We discuss separately the three cases $\rho=0, \rho>0$ and $\rho<0$.

THE SUBCASE $k=1, r=n \geq 2$ AND $\rho=0$.

With $\rho=0$ denote

$$
c_{1}=\epsilon\left(1+c_{2}^{2}+\ldots+c_{n}^{2}\right)^{1 / 2}
$$

with $\epsilon= \pm 1$. Suppose $\epsilon=1$. Then $\left(-c_{1}, c_{2}, \ldots, c_{n}\right)$ is in $-\Omega$ and for $p \geq(n-2) / 2$ the measure $e^{-c_{1} x_{1}+c_{2} x_{2}+\ldots+c_{n} x_{n}} \nu_{p}(d x)$ is bounded, with Laplace transform

$$
\left(\left(s_{1}-c_{1}\right)^{2}-\left(s_{2}+c_{2}\right)^{2}-\ldots-\left(s_{n}+c_{n}\right)^{2}\right)^{-p} .
$$

This shows that

$$
e^{-c_{1} x_{1}+c_{2} x_{2}+\ldots+c_{n} x_{n}} \nu_{p}(d x)
$$

is the desired probability. This probability is a Wishart distribution on the cone $\Omega$ in the sense of Jordan algebras (see Faraut and Koránvi (1994), Jensen (1988), Massam (1994) or Casalis and Letad (1994)). If $\epsilon=-1$ we take its image by 
$x \mapsto-x$. If $p<(n-2) / 2$ it is easy to use the Gindikin Theorem to see that the probability $\mu_{p, c, v}$ does not exist.

THE SUBCASE $k=1, r=n \geq 2$ AND $\rho>0$.

Assume first that $p \geq(n-2) / 2$. Suppose that $c_{1}>0$. The fact that $\rho>0$ implies that $\left(-c_{1}, c_{2}, \ldots, c_{n}\right)$ is in $-\Omega_{\rho}$, and the measure on $\bar{\Omega}$ defined by $e^{-c_{1} x_{1}+c_{2} x_{2}} \nu_{p, \rho}(d x)$ is bounded and has the Laplace transform

$$
\left(\left(s_{1}-c_{1}\right)^{2}-\left(s_{2}+c_{2}\right)^{2}-\ldots-\left(s_{n}+c_{n}\right)^{2}-\rho\right)^{-p}=(1-2\langle c, s\rangle+v(s))^{-p} .
$$

This shows that $e^{-c_{1} x_{1}+c_{2} x_{2}+\ldots+c_{n} x_{n}} \nu_{p, \rho}(d x)$ is the desired probability $\mu_{p, c, v}$. If $c_{1}<0$ we consider its image by $x \mapsto-x$ for getting $\mu_{p, c, v}$.

Recall that $\rho>0$. We now assume $p<(n-2) / 2$, and we prove that $\mu_{p, c, v}$ does not exist. Suppose that $\mu_{p, c, v}$ exists. For any integer $N$ we have $\mu_{p, c, v}^{* N}=\mu_{N p, c, v}$. Since $\mu_{N p, c, v}$ is concentrated on the closed convex cone $\bar{\Omega}$ for $N$ large enough by the previous analysis, $\mu_{p, c, v}$ is also concentrated on $\bar{\Omega}$.

We now consider two polynomials

$$
v(\theta)=\theta_{1}^{2}-\theta_{2}^{2}-\ldots-\theta_{n}^{2}, \quad \Delta(\theta)=1-2\langle c, \theta\rangle+v(\theta) .
$$

Observe that if $x$ and $x^{\prime}$ are in the cone $\Omega$ defined by (3.23), the Schwarz inequality applied to $\left(x_{2}, \ldots, x_{n}\right)$ and $\left(x_{2}^{\prime}, \ldots, x_{n}^{\prime}\right)$ shows that $v\left(x+x^{\prime}\right)>v(x)+v\left(x^{\prime}\right)$. Since

$$
\Omega \supset \Omega_{\rho}=\left\{x \in \mathbb{R}^{n} ; x_{1}>0, v(x)>\rho\right\}
$$

the inequality $v\left(x+x^{\prime}\right)>v(x)+v\left(x^{\prime}\right)$ holds on $\Omega_{\rho}$. This shows that $\Omega_{\rho}$ is an additive semigroup, that is, $x+x^{\prime}$ is in $\Omega_{\rho}$ if $x$ and $x^{\prime}$ are in $\Omega_{\rho}$. We apply it to $x=-\theta$ and $x^{\prime}=c$ to obtain that $\Delta$ is positive on $-\Omega_{\rho}$ :

$$
\Delta(\theta)=1-2\langle c, \theta\rangle+v(\theta)=v(-\theta+c)-v(c)+1=v(-\theta+c)-\rho>0 .
$$

We now apply the operator $v\left(\frac{\partial}{\partial \theta}\right)$ to the two members of the following identity:

$$
(\Delta(\theta))^{-p}=\int_{\bar{\Omega}} e^{\langle\theta, x\rangle} \mu_{p, c, v}(d x) .
$$

This identity is true for all $\theta$ in $-\Omega_{\rho}$ since $\Delta$ is positive there. We get

$$
v\left(\frac{\partial}{\partial \theta}\right)(\Delta(\theta))^{-p}=\int_{\bar{\Omega}} e^{\langle\theta, x\rangle} v(x) \mu_{p, c, v}(d x) .
$$

Now a thorough and standard computation of the left hand side of (3.33) shows that it is equal to $4(\rho+\Delta(\theta))(\Delta(\theta))^{-p-2} p\left(p-p_{0}(\theta)\right)$, where

$$
p_{0}(\theta)=\frac{(n-2) \Delta(\theta)-2 \rho}{2(\Delta(\theta)+\rho)}<\frac{n-2}{2} .
$$

Note that $\rho+\Delta(\theta)$ is positive on $-\Omega_{\rho}$. Furthermore $\rho+\Delta(\theta)$ does not depend on $p$. Since the right hand side of (3.33) is non-negative the existence of $\mu_{p, c, v}$ would imply $p \geq p_{0}(\theta)$ for all $\theta \in-\Omega_{\rho}$. Since $\Delta$ is unbounded on $-\Omega_{\rho}$, this implies that $p \geq(n-2) / 2$, which contradicts our assumption.

THE SUBCASE $k=1,2 \leq r=n$ AND $\rho<0$.

With $\rho<0$ and $p \geq(n-2) / 2$ we now prove that $\mu_{p, c, v}$ does not exist. If not, standard reasoning shows that there would exist a positive measure $\nu_{p, \rho}$ such that either (3.26) or (3.27) hold (according to $p>(n-2) / 2$ or $p=(n-2) / 2)$ : for this compute the Laplace transforms of the right hand sides of (3.26) or (3.27). But the 
function $x \mapsto f_{p}(\rho v(x))$ is not positive when $\rho<0$. To see this we relate $f_{p}$ to the classical Bessel function $J_{p}$ by the formula

$$
\left(\frac{z}{2}\right)^{p-1} f_{p}\left(-\frac{1}{4} z^{2}\right)=J_{p-1}(z)
$$

which implies (see Watson (1966) Jensen (1988), Massam (1994) or Casalis and Letac (1994)), chapter XV) that $f_{p}$ has an infinity of simple zeros on $(-\infty, 0)$ (and no other zeros in the complex plane).

Finally, we show that $k=1$ and $0<p<(n-2) / 2$ implies that $\mu_{p, c, v}$ does not exist. The statement is clear: since $\rho<0$, the existence of $\mu_{p, c, v}$ would imply the existence of $\mu_{N p, c, v}$ for any integer $N$. But we have already seen that $\mu_{p, c, v}$ does not exist for $\rho<0$ and $p>(n-2) / 2$.

THE CASE $k=1$ AND $1 \leq r<n$.

In this case, if $c_{r+1}=\ldots=c_{n}=0$ the problem of the existence of $\mu_{p, c, v}$ is solved by the previous cases $k=1, r=n \geq 2$ or $k=1=n$. If $c_{i} \neq 0$ for some $i$ with $r<i \leq n$, we prove that $\mu_{p, c, v}$ does not exist. To see this, we observe that $\left(1-2 c_{1} s_{1}+s_{1}^{2}-2 c_{2} s_{2}\right)^{-p}$ is not a Laplace transform of the distribution of a random variable $\left(X_{1}, X_{2}\right)$. For in this case we have $\mathbb{E}\left(e^{t\left(X_{1}-X_{2} c_{1} / c_{2}\right)}\right)=\left(1+t^{2}\right)^{-p}$ which is impossible since $t \mapsto\left(1+t^{2}\right)^{-p}$ is not a convex function around 0 . More generally the following function defined in a neighborhood of zero by

$$
\left(1-2 c_{1} s_{1}+s_{1}^{2}-2 c_{2} s_{2}-s_{2}^{2}-\ldots-s_{r}^{2}-2 c_{r+1} s_{r+1}-\ldots-2 c_{n} s_{n}\right)^{-p}
$$

cannot be the Laplace transform of a probability if $\left(c_{r+1}, \ldots, c_{n}\right) \neq 0$. If $c_{r+1} \neq 0$ (say), then inserting $s_{i}=0$ for $i \neq 1$ and $i \neq r+1$ brings us back to the preceding impossible case.

\section{LAPLACE TRANSFORMS $\left(f_{1}\left(s_{1}\right)-s_{2}\right)^{-p}$}

Our next task is to study the distributions $\mu_{p, f_{1}}$ in $\mathbb{R}^{2}$ (essentially) which occur in Theorem 2.1 for $r=1$ and $a^{2}<b<a$, with Laplace transform $\left(f_{1}\left(s_{1}\right)-s_{2}\right)^{-p}$. Actually, replacing $\mathbb{R}^{2}$ by $\mathbb{R}^{m+1}$ is rather natural. Note that since $\left(s_{1}, s_{2}\right) \mapsto$ $\left(f_{1}\left(s_{1}\right)-s_{2}\right)^{-p}$ is a Laplace transform, then it must be convex, thus $f_{1}$ must be concave. Furthermore, the domain of definition of a Laplace transform is convex, thus the interior of the domain of definition of $f_{1}$ must be an open convex subset $C$ of $\mathbb{R}^{m}$ and the interior of the domain of definition of $\left(s_{1}, s_{2}\right) \mapsto\left(f_{1}\left(s_{1}\right)-s_{2}\right)^{-p}$ is $\left\{\left(s_{1}, s_{2}\right) \in C \times \mathbb{R} ; f_{1}\left(s_{1}\right)-s_{2}>0\right\}$. In order to avoid difficulties about continuity at the boundary of the Laplace transforms, we prefer to work with the interior of the domain of definition of Laplace transforms rather than with the domain itself. Recall that the Laplace transform of a probability is not continuous in general at one of the boundary points: continuity holds only along straight lines containing the boundary point (an example due to Hoffmann-Jorgensen can be found in Barndorff-Nielsen (1978), page 104).

Proposition 4.1. Let $f_{1}$ be a concave function defined on some convex open subset $C$ of $\mathbb{R}^{m}$ containing 0 in its closure and let $p>0$. A necessary and sufficient condition for the existence of a probability $\mu_{p, f_{1}}$ on $\mathbb{R}^{m+1}$ with Laplace transform $\left(f_{1}\left(s_{1}\right)-s_{2}\right)^{-p}$ is the existence of some infinitely divisible distribution $\left(\nu_{t}\right)_{t>0}$ on $\mathbb{R}^{m}$ such that the Laplace transform of $\nu_{t}$ is $\exp t\left(1-f_{1}\left(s_{1}\right)\right)$. Under these circumstances

$$
\mu_{p, f_{1}}(d x, d t)=\nu_{t}(d x) \gamma_{p, 1}(d t) .
$$


That means that if $(Y(t))_{t \geq 0}$ is a Lévy process on $\mathbb{R}^{m}$ governed by $\left(\nu_{t}\right)_{t>0}$ and if $T$ is an independent random variable with distribution $\gamma_{p, 1}(d t)$, then $\mu_{p, f_{1}}$ is the distribution in $\mathbb{R}^{m+1}$ of $(Y(T), T)$.

Proof. The part $\Leftarrow$ is standard. In order to prove the converse $\Rightarrow$ we consider a random variable $(X, T) \sim \mu_{p, f_{1}}(d x, d t)$ in $\mathbb{R}^{m} \times \mathbb{R}$ such that on the set

$$
D=\left\{\left(s_{1}, s_{2}\right) ; s_{1} \in C, s_{2}<f_{1}\left(s_{1}\right)\right\}
$$

we have

$$
\mathbb{E}\left(e^{\left\langle s_{1}, X\right\rangle+s_{2} T}\right)=\left(f_{1}\left(s_{1}\right)-s_{2}\right)^{-p} .
$$

Since $\mu_{p, f_{1}}$ is a probability, $(0,0)$ belongs to the closure of $D$, and therefore $\lim _{s_{1} \rightarrow 0} f_{1}\left(s_{1}\right)=1$ for radial limit. Thus we can claim that for $s_{2}<1$ we have $\mathbb{E}\left(e^{s_{2} T}\right)=\left(1-s_{2}\right)^{-p}$. In other words the marginal distribution of $T$ must be $\gamma_{p, 1}$, where for $a>0$ we denote $\gamma_{p, a}(d t)=e^{-a t} a^{p} t^{p-1} \mathbf{1}_{(0, \infty)}(t) \frac{d t}{\Gamma(p)}$. Therefore if we denote by $\nu_{t}(d x)$ the conditional distribution of $X$ knowing $T=t$, we get $\mu_{p, f_{1}}(d x, d t)=\nu_{t}(d x) \gamma_{p, 1}(d t)$, and thus on $D$ :

$$
\begin{aligned}
\left(f_{1}\left(s_{1}\right)-s_{2}\right)^{-p} & =\int_{\mathbb{R}^{m}} \int_{0}^{\infty} e^{\left\langle s_{1}, x\right\rangle+s_{2} t} \nu_{t}(d x) \gamma_{p, 1}(d t) \\
& =\int_{0}^{\infty} e^{s_{2} t}\left(\int_{\mathbb{R}^{m}} e^{\left\langle s_{1}, x\right\rangle} \nu_{t}(d x)\right) \gamma_{p, 1}(d t) .
\end{aligned}
$$

Since $\lim _{s_{1} \rightarrow 0} f_{1}\left(s_{1}\right)=1$ there is a non-empty convex subset $C_{1}$ of $C$ on which the concave function $f_{1}$ is positive. For $s_{1} \in C_{1}$ and $s_{2}<f_{1}\left(s_{1}\right)$ we have

$$
\left(\frac{f_{1}\left(s_{1}\right)}{f_{1}\left(s_{1}\right)-s_{2}}\right)^{p}=\int_{0}^{\infty} e^{s_{2} t}\left(\int_{\mathbb{R}^{m}} e^{\left\langle s_{1}, x\right\rangle} \nu_{t}(d x)\right)\left(f_{1}\left(s_{1}\right)\right)^{p} \gamma_{p, 1}(d t) .
$$

Thus for fixed $s_{1} \in C_{1}$ we can claim that

$$
\left(\int_{\mathbb{R}^{m}} e^{\left\langle s_{1}, x\right\rangle} \nu_{t}(d x)\right)\left(f_{1}\left(s_{1}\right)\right)^{p} \gamma_{p, 1}(d t)=\gamma_{p, f_{1}\left(s_{1}\right)}(d t) .
$$

Equating the densities we get that for almost all $t>0$ we have on $C_{1}$ :

$$
\int_{\mathbb{R}^{m}} e^{\left\langle s_{1}, x\right\rangle} \nu_{t}(d x)=e^{t\left(1-f_{1}\left(s_{1}\right)\right)} .
$$

Since the the function on $C_{1}$ defined by $e^{t\left(1-f_{1}\left(s_{1}\right)\right)}$ is the Laplace transform of a probability $\nu_{t}$ for almost all $t>0$, one concludes by continuity that it is the Laplace transform of some probability $\nu_{t}$ for all $t>0$, and $\left(\nu_{t}\right)_{t>0}$ is a convolution semigroup. This ends the proof.

\section{Comments.}

1. Note that in the above proof $C_{1} \neq C$ in general: for example if $f_{1}\left(s_{1}\right)=$ $1-\left\|s_{1}\right\|^{2}$, then $C_{1}$ is the open unit ball and $C$ is the Euclidean space $\mathbb{R}^{m}$.

2. There is nothing special in the gamma distribution in the statement of Proposition 4.1. If $\mathbb{E}\left(e^{\left\langle s_{1}, X\right\rangle+s_{2} T}\right)$ has the form $e^{\kappa_{0}\left(\kappa_{1}\left(s_{1}\right)+s_{2}\right)}$ and if the distribution $\alpha$ of $T$ is concentrated in $[0, \infty)$, consider the conditional distribution $X \mid T=t \sim \nu_{t}(d x)$. Then $\alpha$ almost surely we have

$$
e^{t\left(\kappa_{1}\left(s_{1}\right)-\kappa_{1}(0)\right)}=\int_{\mathbb{R}^{m}} e^{\left\langle s_{1}, x\right\rangle} \nu_{t}(d x) .
$$

If (4.35) holds for $t=1$ (say), then the fact that (4.35) holds $\alpha$ almost surely implies that the Jorgensen set of $\nu_{1}$ contains the support of $\alpha$. 
In particular, if the support of $\alpha$ contains some interval $[0, a]$, then $\nu_{1}$ is infinitely divisible. See also comment 5 in Section 6 .

3. Let us also mention that in Theorem 2.1, the second part of 3 , we have considered $X=\left\langle X, e_{1}\right\rangle+X^{\prime}$ where $X^{\prime}=T c$ is a multiple of a constant vector $c$ orthogonal to $e_{1}$. With the notation of Proposition 4.1, we have $\left\langle X, e_{1}\right\rangle=Y(T)$.

\section{VARIANCE FUNCTIONS $\frac{1}{p} m \otimes m-\varphi(m) M_{v}$}

In this section, we are going to interpret some of the above results in terms of natural exponential families (NEF). Recall some definitions about NEF. Given a positive measure $\mu$ on $\mathbb{R}^{n}$, not necessarily bounded, consider its Laplace transform, namely the function on $\mathbb{R}^{n}$ valued in $[0, \infty]$ defined by

$$
\theta \mapsto L_{\mu}(\theta)=\int_{\mathbb{R}^{n}} e^{\langle\theta, x\rangle} \mu(d x) \leq \infty .
$$

We denote by $D(\mu)$ the set of $\theta \in \mathbb{R}^{n}$ such that $L_{\mu}(\theta)<\infty$. Hölder inequality implies that $D(\mu)$ is a convex set and that $\kappa_{\mu}=\log L_{\mu}$ is a convex function on it. One denotes by $\Theta(\mu)$ the interior of $D(\mu)$. For instance, if $\mu$ is a probability which has exponential moments (that is, there exists $a>0$ such that $\left.\int_{\mathbb{R}^{n}} e^{a\left(\left|x_{1}\right|+\ldots+\left|x_{n}\right|\right)} \mu(d x)<\infty\right)$, then $\Theta(\mu)$ is not empty.

Let us assume that $\Theta(\mu)$ is not empty. If furthermore $\mu$ is not concentrated on some affine hyperplane, then $\kappa_{\mu}$ is strictly convex and real-analytic on $\Theta(\mu)$. This implies that its differential $\kappa_{\mu}^{\prime}$ (that we consider as taking its values in $\mathbb{R}^{n}$ ) is one-toone. Denote the image of $\Theta(\mu)$ by the function $\kappa_{\mu}^{\prime}$ as the open subset $M(\mu) \subset \mathbb{R}^{n}$. Denote also by $m \mapsto \theta=\psi_{\mu}(m)$ the inverse of $\theta \mapsto m=\kappa_{\mu}^{\prime}(\theta)$. The NEF $F=F(\mu)$ generated by $\mu$ is the set of probabilities $F(\mu)=\{P(\theta, \mu) ; \theta \in \Theta(\mu)\}$ where

$$
P(\theta, \mu)(d x)=e^{\langle\theta, x\rangle-\kappa_{\mu}(\theta)} \mu(d x) .
$$

Note that $F=F(\mu)=F\left(\mu_{1}\right)$ does not imply $\mu=\mu_{1}$, but only the existence of $(a, b) \in \mathbb{R}^{n+1}$ such that $\mu_{1}(d x)=e^{\langle a, x\rangle+b} \mu(d x)$. In this case $\Theta\left(\mu_{1}\right)=a+\Theta(\mu)$ but $M\left(\mu_{1}\right)=M(\mu)$, and we would rather denote it by $M(F)$. Note also that any element of $F$ generates $F$, although the converse is not true: $F=F(\mu)$ does not necessarily imply that $\mu \in F$. The Laplace transform of $P(\theta, \mu)$ is $s \mapsto \frac{L_{\mu}(\theta+s)}{L_{\mu}(\theta)}$ and is defined for $s \in \Theta(\mu)-\theta$.

It is easily seen that $m=\kappa_{\mu}^{\prime}(\theta)=\int_{\mathbb{R}^{n}} x P(\theta, \mu)(d x)$. For this reason $M(F)$ is called the domain of the means of $F$. The Hessian matrix $\kappa_{\mu}^{\prime \prime}(\theta)$ is the covariance matrix of $P(\theta, \mu)$. Therefore, for $m \in M(F)$ the symmetric matrix $V_{F}(m)=$ $\kappa_{\mu}^{\prime \prime}\left(\psi_{\mu}(m)\right)$ is the covariance matrix of the unique element $P(m, F)$ of $F$ with mean $m$. The function defined on $M(F)$ by $m \mapsto V_{F}(m)$ and valued among the symmetric positive definite $(n, n)$ matrices is called the variance function of $F$. Because of the relation $V\left(k_{\mu}^{\prime}(\theta)\right)=\kappa_{\mu}^{\prime \prime}(\theta)$ the variance function determines $k_{\mu}$ up to constants $(a, b) \in \mathbb{R}^{n+1}$, and thus $\left(V_{F}, M(F)\right)$ determines $F$. Finally, we mention that if we take the image $G$ of an NEF $F$ given by $\left(V_{F}, M(F)\right)$ by some affine transformation $x \mapsto A(x)+b$ of $\mathbb{R}^{n}$ where $b \in \mathbb{R}^{n}$ and $A$ is an invertible $n \times n$ matrix, then $M_{G}=A M(F)+b$ and

$$
V_{G}(m)=A V_{F}\left(A^{-1}(m-b)\right) A^{T} .
$$


In this section we are going to find all NEF's $F$ such that there exists a quadratic form $v$, a positive number $p$ and a real function $\varphi$ on $M(F)$ such that $V_{F}(m)=$ $\frac{1}{p} m \otimes m-\varphi(m) M_{v}$ on $M(F)$, where $M_{v}$ is the representative matrix of the quadratic form $v$. As an example, for $v(x)=x_{1}^{2}-x_{2}^{2}-\ldots-x_{n}^{2}$ the family $F_{p}$ of Wishart distributions with the shape parameter $p \geq(n-2) / 2$ on the Lorentz cone $\Omega=\{x \in$ $\left.\mathbb{R}^{n} ; x_{1}>0, v(x)>0\right\}$ has this property. If $P$ denotes the quadratic representation of the Jordan algebra associated to $\Omega$ in the sense of Faraut and Koránvi (1994), page 32, then the variance function is $P(m) / p$. Surprisingly we have on $\Omega$

$$
V_{F_{p}}(m)=\frac{1}{p} P(m)=\frac{1}{p} m \otimes m-\frac{1}{4 p}\left(m_{1}^{2}-m_{2}^{2}-\ldots-m_{n}^{2}\right) \operatorname{Diag}(1,-1, \ldots,-1) .
$$

Note that from (5.36) the image $G$ of an NEF $F$ with $V_{F}(m)=m \otimes m / p-$ $\varphi(m) M_{v}$ by the linear automorphism $x \mapsto A x$ has a variance function of the same form, namely

$$
V_{G}(m)=\frac{1}{p} m \otimes m-\varphi\left(A^{-1} m\right) A M_{v} A^{T} .
$$

This remark will considerably simplify the presentation of our results, since it allows us by proper choice of $A$ to assume that $M_{v}$ has the simple form

$$
M_{v}=\left[\begin{array}{ccc}
I_{k} & 0 & 0 \\
0 & -I_{r-k} & 0 \\
0 & 0 & 0
\end{array}\right]
$$

Theorem 5.1. Let $v$ be a quadratic form on $\mathbb{R}^{n}$ with representative matrix $M_{v}$ having rank $r$ and signature $(k, r-k, n-r)$. Let $p>0$. We assume $n \geq 2$. Let $M$ be a connected open set of $\mathbb{R}^{n}$ and $\varphi$ a real function defined on $M$ such that $m \mapsto \frac{1}{p} m \otimes m-\varphi(m) M_{v}$ is the variance function $V_{F}$ of some NEF $F$ whose domain of the means is $M=M(F)$.

Then $\varphi$ is real analytic on $M$, has no zeros and has constant sign. Assuming $\varphi>0$, then either $k=1, r=n$, or $k=0, r=n$, or $k=0, r=n-1$ or $k=r=1, n=2$. Except when $k=r=1, n=2$ there exist some $c \in \mathbb{R}^{n}$ and some real $\lambda>0$ such that the NEF $F$ is generated by a probability $\mu_{p, c, \lambda v}$ with Laplace transform $\Delta(s)^{-p}$ with $\Delta(s)=1-2\langle c, s\rangle+\lambda v(s)$. Every element $P\left(\theta ; \mu_{p, c, \lambda v}\right)$ of $F$ has the form $\mu_{p, c_{\theta}, \lambda_{\theta} v}$ with $c_{\theta}=\left(c-M_{v} \theta\right) / \Delta(\theta)$ and $\lambda_{\theta}=\lambda / \Delta(\theta)$.

1. If $k=1, r=n$ the vector $c$ must satisfy $\rho=\frac{v^{-1}(c)}{\lambda}-1 \geq 0$. The domain of the means is $M(F)=\Omega_{\rho}$ defined by (3.25). If $\rho>0$ the proportionality factor $\varphi(m)>0$ is

$$
\varphi(m)=\frac{p \lambda}{2 \rho}\left(\left[1+\rho \frac{v^{-1}(m)}{p^{2} \lambda}\right]^{1 / 2}-1\right) .
$$

If $\rho=0$ the natural exponential family $F$ is the family of Wishart distributions on the Lorentz cone $\Omega$ associated to $v$, with the shape parameter $p \geq(n-2) / 2$. In this case $\varphi(m)=\frac{v^{-1}(m)}{4 p}$.

2. If $k=0, r=n$ the domain of the means is $\mathbb{R}^{n}$. With the Euclidean structure $\|x\|^{2}=-v^{-1}(x)$ we have

$$
\varphi(m)=\frac{p}{\lambda\left(\|c\|^{2}+\lambda\right)}\left[\left(\lambda^{2}+\left(\|c\|^{2}+\lambda\right)\|m\|^{2}\right)^{1 / 2}+\lambda\right] .
$$


3. If $k=0, r=n-1$ we take for simplicity $v(x)=v^{-1}(x)=-x_{1}^{2}-\ldots-x_{n-1}^{2}$. Then $c_{n} \neq 0$ and we have $\varphi(m)=\frac{\lambda m_{n}}{2 c_{n}}$, and the domain of the means is $\mathbb{R}^{n-1} \times(0, \infty)$ if $c_{n}>0$ and $\mathbb{R}^{n-1} \times(-\infty, 0)$ if $c_{n}<0$.

4. In the exceptional case $k=r=1, n=2$ the NEF is generated by a probability measure $\mu_{p, f_{1}}(d x, d t)=\nu_{t}(d x) \gamma_{p, 1}(d t)$ as defined in Section $I V$. Denote by $F_{1}$ the $N E F$ in $\mathbb{R}$ generated by $\nu_{1}$, denote by $(a, b)$ the domain of the means of $F_{1}$ and denote by $V_{F_{1}}$ its variance function. Then the domain of the means of $F$ is the cone

$$
\begin{gathered}
\left\{\left(m_{1}, m_{2}\right) ; m_{2}>0, a m_{2}<m_{1}<b m_{2}\right\} \\
\text { and } \varphi\left(m_{1}, m_{2}\right) \text { is }-m_{2} V_{F_{1}}\left(m_{1} / m_{2}\right) . \text { Consequently } \\
V_{F}\left(m_{1}, m_{2}\right)=\left[\begin{array}{cc}
\frac{m_{1}^{2}}{p}+m_{2} V_{F_{1}}\left(\frac{m_{1}}{m_{2}}\right) & \frac{m_{1} m_{2}}{p} \\
\frac{m_{1} m_{2}}{p} & \frac{m_{2}^{2}}{p}
\end{array}\right] .
\end{gathered}
$$

Proof. The quadratic form $v$ cannot be 0 since the rank of $V_{F}(m)$ is always $n \geq 2$. Since a variance function is real analytic and since $v \neq 0$, the real analyticity of $\varphi$ is obvious. If $\varphi\left(m_{0}\right)=0$ for some $m_{0} \in M$, then the rank of $V\left(m_{0}\right)$ is $1 \neq n$, a contradiction. Since $M$ is connected, $\varphi$ has constant sign. Without loss of generality, we choose in the sequel $\varphi(m)>0$. If not, we change $v$ into $-v$.

Since $n=\operatorname{rank} V_{F}(m) \leq 1+r$ we get $r \geq n-1$. If $r=n$

$$
V_{F}(m)=\frac{1}{p} m \otimes m+\left[\begin{array}{cc}
-\varphi(m) I_{k} & 0 \\
0 & \varphi(m) I_{n-k}
\end{array}\right] .
$$

Clearly since $\varphi(m)>0$, this matrix is positive definite only if $k \leq 1$ : just watch the first $(k, k)$ block of the matrix $V_{F}(m)$.

For $r=n-1$ we consider the matrix

$$
V_{F}(m)=\frac{1}{p} m \otimes m+\left[\begin{array}{ccc}
-\varphi(m) I_{k} & 0 & 0 \\
0 & \varphi(m) I_{n-1-k} & 0 \\
0 & 0 & 0
\end{array}\right] .
$$

Similarly as above we see that $k \leq 1$. However, $k=1$ is excluded by considering the $(2,2)$ submatrix obtained with the first and last rows and columns.

THE CASE $k=1, r=n$.

Let $\mu$ be a generating measure of $F$ and write $\kappa$ as the $\log$ of the Laplace transform of $\mu$ defined on the open convex set $\Theta$. Then $s \mapsto \kappa^{\prime}(s)$ is a bijective mapping from $\Theta$ on $M$. Since $\varphi$ has no zeros, we denote $f(s)=\frac{1}{\varphi\left(\kappa^{\prime}(s)\right)}$. Thus we have

$$
\kappa^{\prime \prime}(s)=\frac{1}{p} \kappa^{\prime}(s) \otimes \kappa^{\prime}(s)-\frac{1}{f(s)} v .
$$

At this point we can use the discussion made after (2.11) and claim that since $r \geq 2$ there exists $c \in \mathbb{R}^{n}$ and $\lambda>0$ such that $f$ has the form (2.16) and $\mu=\mu_{p, c, \lambda v}$. This implies that the domain of the means $M$ is the Lorentz cone $\Omega$ and that the domain of the Laplace transform $\Theta$ is $-\Omega_{\rho}$, where $\rho=\frac{v^{-1}(c)}{\lambda}-1 \geq 0$ (by Theorem 3.1 , part 3 ) and $\Omega_{\rho}$ is defined by (3.25).

The only thing left to do is to compute $\varphi(m)$. We use $M_{v}$ as the representative matrix of $v$. As usual, the quadratic form associated to $M_{v}^{-1}$ is denoted $v^{-1}$. Since 
$\kappa(s)=-p \log \Delta$ with $\Delta=1-2\langle c, s\rangle+\lambda v(s)$, we write $m=\kappa^{\prime}(s)=\frac{2 p}{\Delta}\left(c-\lambda M_{v} s\right)$. Denote by $\psi: M \rightarrow \Theta$ the inverse function of $\kappa^{\prime}$. Thus

$$
s=\psi(m)=\frac{1}{\lambda} M_{v}^{-1} c-\frac{\Delta}{2 \lambda p} M_{v}^{-1} m .
$$

We carry this in the definition of $\Delta(s)$ for computing $\Delta$ :

$$
\begin{aligned}
\Delta & =1-\frac{2}{\lambda}\left\langle c, M_{v}^{-1} c\right\rangle+\frac{\Delta}{\lambda p}\left\langle c, M_{v}^{-1} m\right\rangle+\frac{1}{\lambda} v\left(M_{v}^{-1} c-\frac{\Delta}{2 p} M_{v}^{-1} m\right) \\
& =1-\frac{1}{\lambda} v^{-1}(c)+\frac{\Delta^{2}}{4 \lambda p^{2}} v^{-1}(m)=-\rho+\frac{\Delta^{2}}{4 \lambda p^{2}} v^{-1}(m) .
\end{aligned}
$$

In order to solve this second degree equation in $\Delta$ we denote

$$
\delta=\left[1+\rho \frac{v^{-1}(m)}{\lambda p^{2}}\right]^{1 / 2}
$$

Introducing $\epsilon= \pm 1$ gives $\Delta=\frac{2 \lambda p^{2}}{v^{-1}(m)}(1+\epsilon \delta)$, and finally for $\rho>0$ we get

$$
\varphi(m)=\frac{1}{f(\psi(m))}=\frac{\lambda p}{\Delta}=\frac{\lambda p}{2} \frac{\epsilon \delta-1}{\rho} .
$$

In order to decide whether $\epsilon=1$ or not, we observe that $-\varphi(m) v$ must have at least $n-1$ positive signs in its signature. Since $k=1$ and $r=n$, this implies $\varphi(m)>0$ and $\epsilon=1$. For $\rho=0$ an easy discussion shows that $\varphi(m)=\frac{v^{-1}(m)}{4 p}$.

THE CASE $k=0, r=n$.

The discussion is rather similar, and changes occur only when finding the sign of $\epsilon$ for choosing the correct root of the second degree equation giving $\Delta$. We skip the details.

THE CASE $k=0, r=n-1$.

For $n=2$ by the discussion given in the proof of Theorem 2.1, case $a^{2}<b<a$, $r=1$ (the second part where $g$ is not identically zero), we get that $\Delta(s)=1-$ $2 c_{1} s_{1}-\lambda s_{1}^{2}-2 c_{2} s_{2}$. For $n>2$ we have $\Delta(s)=1-2\langle c, s\rangle-\lambda\left(s_{1}^{2}+\ldots+s_{n-1}^{2}\right)$. Thus in both cases $n=2$ and $n>2$ we are sent back to a similar discussion as in the case $k=1$ and $r=n$. Thus $\varphi(m)=p \lambda / \Delta$. Moreover

$$
m_{n}=\frac{\partial \kappa}{\partial s_{n}}=\frac{2 p c_{n}}{\Delta} .
$$

Combining the two formulas we get $\varphi(m)=\frac{\lambda m_{n}}{2 c_{n}}$.

The EXCEPTIONAL CASE $k=r=1, n=2$.

We have seen in Section 2 that the family is generated by a probability $\mu_{p, f_{1}}$ on $\mathbb{R}^{2}$ with Laplace transform $\left(f_{1}\left(s_{1}\right)-s_{2}\right)^{-p}$. By analyticity, this Laplace transform is defined on

$$
\Theta=\left\{\left(s_{1}, s_{2}\right) ; s_{1} \in I, s_{2}<f_{1}\left(s_{1}\right)\right\},
$$

where $I$ is the open domain of the definition of $f_{1}$. Since this domain is convex, this implies that $I$ is an open interval and that $f_{1}$ is concave. Furthermore $0 \in \bar{I}$ 
and $\lim _{s_{1} \rightarrow 0} f_{1}\left(s_{1}\right)=1$. The cumulant function is $k=-p \log f$ where $f\left(s_{1}, s_{2}\right)=$ $f_{1}\left(s_{1}\right)-s_{2}$. Thus

$$
m_{1}=-p \frac{f_{1}^{\prime}\left(s_{1}\right)}{f}, \quad m_{2}=p \frac{1}{f} .
$$

This implies that $f_{1}^{\prime}\left(s_{1}\right)=-m_{1} / m_{2}$. Observe that since $k$ is strictly convex, then $\left(s_{1}, s_{2}\right) \mapsto f_{1}\left(s_{1}\right)-s_{2}$ is strictly concave and thus $s_{1} \mapsto f_{1}\left(s_{1}\right)$ is strictly concave, too. Hence $s_{1} \mapsto f_{1}^{\prime}\left(s_{1}\right)=-m_{1} / m_{2}$ is one to one and $s_{1}$ is a function of $m_{1} / m_{2}$. Since the cumulant function of $F_{1}$ is $1-f_{1}\left(s_{1}\right)$, the domain of the means of $F_{1}$ is $(a, b)=-f_{1}^{\prime}(I)$. We now check that the map from $\Theta$ to $(a, b) \times(0, \infty)$ is bijective: for any $(c, y) \in(a, b) \times(0, \infty)$ there exists a unique $s_{1} \in I$ such that $c=-f_{1}^{\prime}\left(s_{1}\right)$ and a $s_{2}<f\left(s_{1}\right)$ such that $y=\frac{p}{f\left(s_{1}\right)-p}$. Thus the domain of the means is as claimed.

Since $\frac{\partial^{2} k}{\partial s_{1}^{2}}=\frac{m_{1}^{2}}{p}-m_{2} f_{1}^{\prime \prime}\left(s_{1}\right)=\frac{m_{1}^{2}}{p}-\varphi\left(m_{1}, m_{2}\right)$ and since $s_{1}$ is a function of $m_{1} / m_{2}$ we get that $\varphi\left(m_{1}, m_{2}\right)=m_{2} \varphi_{1}\left(m_{1} / m_{2}\right)$, where $\varphi_{1}$ is defined on $(a, b)$. Since $V_{F_{1}}\left(-f_{1}^{\prime}\left(s_{1}\right)\right)=-f_{1}^{\prime \prime}\left(s_{1}\right)$ we can claim that $\varphi_{1}=-V_{F_{1}}$, and this ends the proof.

\section{Comments And EXAmples}

1. Examples. We concentrate on the case $a^{2}<b<a$ and $n=2$. Up to linear changes we can assume that the possible representative matrix $M_{v}$ of $v$ is

$$
B=\left[\begin{array}{ll}
1 & 0 \\
0 & 0
\end{array}\right],-I_{2}=\left[\begin{array}{cc}
-1 & 0 \\
0 & -1
\end{array}\right], W=\left[\begin{array}{ll}
0 & 1 \\
1 & 0
\end{array}\right] .
$$

To see this we observe that $(2,0,0),(1,1,0),(0,2,0),(1,0,1)$, and $(0,1,1)$ are the possible signatures $(k, r-k, n-r)$ for a non-zero $v$, and $n=2$. However cases $(2,0,0)$ and $(0,2,0)$ coincide (corresponding to $\left.-I_{2}\right)$ as well as $(1,0,1)$ and $(0,1,1)$ (corresponding to $B$ ) since $Q_{v}=Q_{-v}$ : see the comments at the beginning of Section 3. Cases $B$ and $W$ have been covered in Bobecka and Wesolowski (2004) and Wang (1981). The case $-I_{2}$ is new and is described by (3.30).

2. Exponential moments. Wang's theorem is for positive random variables: they do not necessarily have exponential moments, but they have non-trivial Laplace transforms, and this is equivalent after a trivial change of the probability measures of $X$ and $Y$. In Theorem 2.1, we do not make a hypothesis about positiveness, but only about exponential moments. One can wonder if the hypothesis could be reduced to the existence of the first two moments. There is apparently serious difficulty in deducing from the analog of (2.9), written in terms of Fourier transforms which holds only for all $q \in Q_{v}$, the fact that all higher derivatives of the Fourier transform of $X$ do exist. This problem does not exist in the one dimensional cases as considered by Lukacs (1955).

3. Simple quadratic families. In a celebrated paper Casalis (1996) has characterized all the natural exponential families on $\mathbb{R}^{n}$ (called simple quadratic families, or Casalis families) such that their variance functions have the form

$$
V_{F}(m)=a m \otimes m+B(m)+C,
$$

where $m \mapsto B(m)$ is linear, $C$ is a constant matrix and $a$ is a real number. If $a>0, C=0$ and $B(m)$ is the product of a constant matrix $M_{v}$ by a linear form $m \mapsto \varphi(m)$, we get the simple quadratic families covered by our Section 5 . If $n \geq 3$, among these $2 n+4$ Casalis families, only one has this property, namely her gamma family of type 1 which coincides with case 3 of Theorem 5.1. If $n=2$ two of the 
8 Casalis families appear in the exceptional case 4 of Theorem 5.1 by taking $F_{1}$ either as a normal family or a Poisson family.

4. Diagonal variance families. In Bar-Lev et al. (1994) all the natural exponential families on $\mathbb{R}^{n}$ such that their variance functions satisfy

$$
\operatorname{Diag}\left(V_{F}(m)\right)=\left(f_{1}\left(m_{1}\right), \ldots, f_{n}\left(m_{n}\right)\right)
$$

are characterized and called diagonal variance families. When $v \neq 0$ and $M_{v}$ has zero diagonal, our natural exponential families are of the diagonal variance type. If $n=2$ we are in the Wang case. For $n \geq 3$ clearly $k=r$ or $k=0$ is impossible, since it implies that $M_{v}$ or $-M_{v}$ would be a semipositive definite matrix, which is impossible if its diagonal is 0 . An example of the case where $k=1$ and $r=n \geq 3$ is $M_{v}=I_{n}-J_{n}$, where $J_{n}$ is the matrix of ones. For instance for $n=3$

$$
M_{v}=\left[\begin{array}{ccc}
0 & -1 & -1 \\
-1 & 0 & -1 \\
-1 & -1 & 0
\end{array}\right] \text {. }
$$

It is easily seen that the characteristic polynomial of $M_{v}$ is

$$
(-1)^{n}(\lambda-n+1)(\lambda+1)^{n-1},
$$

thus the signature is $(1, n-1,0)$.

This example is rather interesting since Theorem 5.1 provides the value of $\varphi(m)$ (part 1). Here $v(m)=\sum_{j=1}^{n} m_{j}^{2}-\left(\sum_{j=1}^{n} m_{j}\right)^{2}$. There are very few cases of diagonal variance families for $n \geq 3$ such that the off diagonal elements can be computed, and the present calculation offers an example of such a situation. Note that for $n \geq 3$ any signature $(k, r-k, n-r)$ can be reached by a symmetric matrix $M_{v}$ with zero diagonal when $0<k<r$.

The diagonal variance families such that the diagonal of the variance function is $\left(m_{1}^{2} / p, \ldots, m_{n}^{2} / p\right)$ have a Laplace transform of the form $P(s)^{-p}$ as in (1.4). Bernardoff (2006) has given the necessary and sufficient conditions of infinite divisibility of such a distribution in $\mathbb{R}^{n}$ under the extra hypothesis that $c_{\{1, \ldots, n\}} \neq 0$. In our case $P(s)$ is a second degree polynomial, thus for $n \geq 3$ this condition $c_{\{1, \ldots, n\}} \neq 0$ cannot be satisfied. Note that Theorem 3.1 shows that our distributions are not infinitely divisible, since $p$ must be $\geq(n-2) / 2$. For $n=2$ we are sent back again to Wang's theorem, where the distributions are infinitely divisible.

5. ExCEPTIOnAL CASE. It is easy to see that the NEF generated on $\mathbb{R}^{m+1}$ by the distribution appearing in (4.35) is simply

$$
\left[\begin{array}{cc}
\frac{V_{0}\left(m_{2}\right)}{m_{2}^{2}} m_{1} \otimes m_{1}+m_{2} V_{1}\left(\frac{m_{1}}{m_{2}}\right) & \frac{m_{1}}{m_{2}} V_{0}\left(m_{2}\right) \\
\frac{m_{1}^{T}}{m_{2}} V_{0}\left(m_{2}\right) & V_{0}\left(m_{2}\right)
\end{array}\right],
$$

where $V_{0}$ and $V_{1}$ are the variance functions of the NEFs generated by $\alpha$ and $\nu_{1}$ (assuming that 1 is in the support of $\alpha$ ).

6. Quadratic REgression for Wishart distributions on Jordan aLGEBRAS. In Letac and Massam (1998) a characterization of Wishart distributions on the symmetric cone $\Omega$ associated to a Euclidean Jordan algebra $E$ based on regression conditions is given: consider the two symmetric operators on $E$,

$$
Q_{1}(x)=d^{\prime} x \otimes x+P(x), \quad Q_{2}(x)=-x \otimes x+P(x),
$$

where $P$ is the quadratic representation of $E$ and $2 d^{\prime}$ is the Peirce constant of $E$. Suppose that $X$ and $Y$ are independent random variables valued in $\bar{\Omega}$ and that 
there exist real numbers $a, b_{1}$ and $b_{2}$ such that $\mathbb{E}(X \mid X+Y)=a(X+Y)$ and

$$
\mathbb{E}\left(Q_{i}(X) \mid X+Y\right)=b_{i} Q_{i}(X+Y)
$$

for $i=1,2$. Then one proves that $X$ and $Y$ are Wishart distributed with the same scale parameter and with shape parameters $p$ and $q$. In this case

$$
b_{1}=\frac{p}{p+q} \times \frac{p+1}{p+q+1}, b_{2}=\frac{p}{p+q} \times \frac{p-d^{\prime}}{p+q-d^{\prime}} .
$$

It is interesting to compare this result with the present paper.

If $S$ is any symmetric operator on $E$, consider the quadratic form on $E$ defined by $x \mapsto q_{i}(S)(x)=$ trace $\left(S Q_{i}(x)\right)$. Denote by $\mathcal{Q}_{i}$ the space of all quadratic forms $q_{i}(S)$ on $V$. Then a reformulation of the above characterization of the Wishart distributions on $V$ is obtained by saying that $\mathbb{E}(q(X) \mid X+Y)=b_{i} q(X+Y)$ for all $q \in \mathcal{Q}_{i}$ and for $i=1,2$ and $\mathbb{E}(X \mid X+Y)=a(X+Y)$ (here $q(x)=q_{i}(S)(x)$ for some symmetric operator $S$ on $E$ ).

The above example suggests that possibly new interesting distributions on a Euclidean space $E$ could be discovered by choosing independent linear subspaces $\mathcal{Q}_{1}, \ldots, \mathcal{Q}_{l}$ of the space of quadratic forms on $E$ such that $\mathbb{E}\left(Q_{i}(X) \mid X+Y\right)=$ $b_{i} Q_{i}(X+Y)$ holds for all $Q_{i} \in \mathcal{Q}_{i}$ with $i=1, \ldots, l$ and some fixed real numbers $b_{1}, \ldots, b_{l}$. The paper by Letac and Massam (1998) was therefore devoted to a particular case where $l=2$. The present paper is devoted to the case $l=1$ where the only restriction for $\mathcal{Q}_{1}$ is to be of codimension 1 (while (1.4) was considering the case where $\mathcal{Q}_{1}$ is the set of $q$ such that $M_{q}$ is diagonal).

\section{REFERENCES}

Bar-Lev, S.K., Bshouty, D., Enis, P., Letac, G., Lu, I. and Richards, D. (1994) The diagonal multivariate natural exponential families and their classification. J. Theor. Probab. 4, 883-929. MR1295545 (96b:60030)

Barndorff-Nielsen, O. (1978) Information and Exponential Families in Statistical Theory, Wiley, Chichester. MR489333(82k:62011)

Bernardoff, P. (2006) Which multivariate gamma distributions are infinitely divisible? Bernoulli 12, 169-189. MR2202328 (2006m:60021)

Bobecka, K. and Wesołowski, J. (2004) Bivariate Lukacs type regression characterizations. J. Appl. Statist. Sci. 13, 49-57. MR2091930 (2005h:62146)

Casalis, M. (1996) The $2 d+4$ simple quadratic natural exponential families on $\mathbb{R}^{d}$. Ann. Statist. 24, 1828-1854. MR.1416663 (97h:60011)

Casalis, M. and Letac, G. (1994) Characterization of the Jorgensen set in the generalized linear model. Test 3, 145-162. MR1293112 (95m:62109)

Faraut, J. and Korányi, A. (1994) Analysis on Symmetric Cones. Oxford University Press, New York. MR:1446489 (98g:17031)

Gindikin, S. (1975) Invariant generalized functions in homogeneous domains. Functional Anal. Appl. 9, 50-52. MR0377423 (51:13595)

Griffiths, R.C. (1984) Characterization of infinitely divisible multivariate gamma distributions. $J$. Multivar. Anal. 15, 13-20. MR755813 (85m:60027)

Jensen, S.T. (1988) Covariance hypotheses which are linear in both the covariance and the inverse covariance. Ann. Statist. 16, 302-322. MR 924873 (88m:62076)

Letac, G. and Massam, H. (1998) Quadratic and inverse regression for Wishart distributions. Ann. Statist. 26, 573-595. MR:1626071 (99f:62071)

Lukacs, E. (1955) A characterization of the gamma distribution. Ann. Math. Statist. 26, 319-324. MR0069408 (16:1034b)

Massam, H. (1994) An exact decomposition theorem and a unified view of some related distributions for a class of exponential transformation models on symmetric cones. Ann. Statist. 22, 369-394. MR 1272089 (95f:62015) 
Watson, G. W. (1966) A Treatise on the Theory of Bessel Functions. University Press, Cambridge. MR1349110 (96i:33010)

Wang, Y. (1981) Extensions of Lukacs' characterization of the gamma distribution. In: Analytic Methods in Probability Theory, Lect. Notes in Math. 861, Springer, New York, 166-177. MR655271 (83m:62029)

Laboratoire de Statistique et Probabilités, Université Paul Sabatier, 31062 TouLOUSE, France

E-mail address: letac@cict.fr

Wydzią Matematyki i Nauk Informacyjnych, Politechnika Warszawska, Warszawa, POLAND

E-mail address: wesolo@mini.pw.edu.pl 